\title{
Covid-19 e diabetes mellitus: uma análise dos vídeos divulgados pela Rede Globo de Televisão
}

\author{
Covid-19 and diabetes mellitus: an analysis of the videos released by Rede \\ Globo de Televisão
}

\section{Covid-19 y diabetes mellitus: un análisis de los videos publicados por Rede Globo de Televisão}

\author{
Jandesson Mendes Coqueiro ${ }^{1, a}$ \\ jandesson.mc@gmail.com | https://orcid.org/0000-0002-5321-5174 \\ ${ }^{1}$ Universidade Federal do Espirito Santo, Departamento de Enfermagem. Vitória, ES, Brasil. \\ a Doutorado em Saúde Coletiva pela Universidade Federal do Espírito Santo.
}

\section{RESUMO}

Estudo qualitativo buscando analisar, a partir do referencial teórico da análise institucional, os vídeos veiculados sobre o diabetes mellitus e a Covid-19 pela mídia televisiva da Rede Globo. Foram identificados 18 vídeos entre 31 de dezembro de 2019 e 31 de maio de 2020. Houve o predomínio de vídeos no mês de março (50\%), médicos e usuários como fonte de informações (55\%), abordagem ao nível de prevenção primária para Covid-19 (72\%) e secundária para o diabetes (89\%) e omissão do Sistema Único de Saúde (100\%). Os vídeos apontaram ausência de medicação para o diabetes nos serviços e a divulgação de informações equivocadas sobre Covid-19. Os profissionais de saúde entrevistados emitiram orientações voltadas para a lógica do risco aos sujeitos com diabetes mellitus. Faz-se necessária maior divulgação das ações do Sistema Único de Saúde em tempos de pandemia Covid-19 e implementação de orientações mais próximas aos modos de viver da população.

Palavras-chaves: Covid-19; Diabetes Mellitus; Pandemias; Televisão; Meios de Comunicação.

\section{ABSTRACT}

Qualitative study seeking to analyze, based on the theoretical framework of institutional analysis, the videos broadcast on diabetes mellitus and Covid-19 by Rede Globo's television media. Eighteen videos were identified between December 31, 2019 and May 31, 2020. There was a predominance of videos in March $(50 \%)$, doctors and users as an information source (55\%), approach to the level of primary prevention for Covid-19 (72\%) and secondary for diabetes (89\%) and omission from the Unified Health System 
(100\%). The videos pointed out the absence of diabetes medication in the services and the dissemination of misinformation about Covid-19. The health professionals interviewed issued guidelines aimed at the logic of risk to subjects with diabetes mellitus. Greater dissemination of the actions of the Unified Health System is necessary in times of pandemic Covid-19 and implementation of guidelines closer to the population's ways of living.

Keywords: Covid-19; Diabetes Mellitus; Pandemics; Television; Communications Media.

\section{RESUMEN}

Estudio cualitativo que busca analizar, con base en el marco teórico del Análisis Institucional, los videos transmitidos sobre diabetes mellitus y Covid-19 por los medios de televisión de Rede Globo. Dieciocho videos fueron identificados entre el 31 de diciembre de 2019 y el 31 de mayo de 2020. Hubo un predominio de videos en marzo (50\%), médicos y usuarios como fuente de información (55\%), enfoque al nivel de prevención primaria para Covid-19 (72\%) y secundaria para diabetes (89\%) y omisión del Sistema Único de Salud (100\%). Los videos señalaron la ausencia de medicamentos para la diabetes en los servicios y la difusión de información errónea sobre Covid-19. Los profesionales de la salud entrevistados emitieron pautas dirigidas a la lógica del riesgo para los sujetos con diabetes mellitus. Es necesaria una mayor difusión de las acciones del Sistema Único de Salud en tiempos de pandemia Covid-19 y la implementación de pautas más cercanas a las formas de vida de la población.

Palabras clave: Covid-19; Diabetes Mellitus; Pandemias; Televisión; Medios de Comunicación.

Este artigo compõe o Dossiê Comunicação, Saúde e Crises Globais.

Contribuição dos autores:

Concepção e desenho do estudo: Jandesson Mendes Coqueiro.

Aquisição, análise ou interpretação dos dados: Jandesson Mendes Coqueiro.

Redação do manuscrito: Jandesson Mendes Coqueiro.

Revisão crítica do conteúdo intelectual: Jandesson Mendes Coqueiro.

Declaração de conflito de interesses: não há.

Fontes de financiamento: não houve.

Considerações éticas: não há.

Agradecimentos/Contribuições adicionais: não há.

Histórico do artigo: submetido: 27 jun. 2020 | aceito: 15 set. 2020. | publicado: 17 dez. 2020.

Apresentação anterior: não há.

Licença CC BY-NC atribuição não comercial. Com essa licença é permitido acessar, baixar (download), copiar, imprimir, compartilhar, reutilizar e distribuir os artigos, desde que para uso não comercial e com a citação da fonte, conferindo os devidos créditos de autoria e menção à Reciis. Nesses casos, nenhuma permissão é necessária por parte dos autores ou dos editores. 


\section{INTRODUÇÃO}

O novo tipo de coronavírus, o SARS-CoV-2, causador da Covid-19, surgiu na China no final de 2019 e alcançou situação de pandemia rapidamente. Tal vírus tem um tropismo pelo sistema respiratório, o que ocasiona desde uma síndrome respiratória aguda, que na maioria dos casos se manifesta de forma leve, com evolução em alguns casos para um tipo grave e célere de pneumonia com insuficiência respiratória importante, progredindo para complicações que podem levar a óbito¹.

Entre os fatores de risco para os desfechos mais graves da Covid-19, como a necessidade de internação em unidade de terapia intensiva (UTI) e/ou uso de ventilação mecânica, estão as seguintes características: idade igual ou superior a 65 anos; doença pulmonar obstrutiva crônica, asma e dependência de oxigênio; problemas cardíacos graves; hipertensão não controlada; diabetes mellitus (DM); doenças cromossômicas ou estado de fragilidade imunológica; insuficiência renal crônica avançada; gestação de alto risco, obesidade grave (IMC > 40); e condições médicas, como doenças hepáticas ${ }^{2,3}$. É possível ainda que a Covid-19 seja uma doença endotelial, podendo levar a quadros graves de coagulopatias e tromboses 4 .

Nesse contexto, cabe ressaltar que as doenças cardiovasculares, respiratórias crônicas, hipertensão arterial, DM, entre outras, são denominadas pela Organização Mundial de Saúde (OMS) 5 doenças crônicas não transmissíveis (DCNT), sendo caracterizadas por etiologias múltiplas, curso prolongado, origem não infecciosa e associadas a incapacidades funcionais e deficiências. Apresentam como fatores de risco aqueles classificados em não modificáveis (herança genética, idade e sexo) e comportamentais (alimentação não saudável, tabagismo, sedentarismo, obesidade e consumo de álcool), além disso, são potencializados pelos aspectos socioeconômicos, culturais e ambientais ${ }^{6}$.

O diabetes mellitus é uma enfermidade caracterizada pelo transtorno metabólico de etiologias diferentes, marcado por aumento da glicemia e distúrbio no metabolismo de carboidratos, proteínas e gorduras resultantes de deficiências da secreção e/ou ação da insulina, ocasionando, quando não controlado, o desenvolvimento de complicações agudas e crônicas e elevadas taxas de morbimortalidade, especialmente na idade adulta ${ }^{7,8}$. Entre as doenças crônicas, o DM tem se tornado relevante para a discussão das comorbidades que aceleraram as complicações da Covid-19, porque o aumento da glicose no sangue pode apresentar uma queda da imunidade no organismo. Além disso, o número de pessoas convivendo com o DM ainda é alto, a saber, no mundo, em 2019, 463 milhões de pessoas conviviam com essa enfermidade; no Brasil, no mesmo ano, verificou-se que mais de 16 milhões de pessoas possuíam essa doença ${ }^{9}$.

Em um estudo realizado com 1.122 sujeitos internados com Covid-19 em 88 hospitais dos Estados Unidos, no período de $1^{0}$ de março a 6 de abril de 2020, foi observado que a taxa de mortalidade de sujeitos com DM e/ou hiperglicemia foi de $28,8 \%$, enquanto que em pessoas sem o DM, ou aqueles que evoluíram durante a internação sem hiperglicemia, foi de 6,2\%. Portanto, a mortalidade nos indivíduos com DM e/ou hiperglicemia foi cinco vezes maior. Outra constatação importante observada neste estudo foi que os sujeitos que evoluíram com hiperglicemia durante a internação, sem diagnóstico prévio de DM, apresentaram índice de mortalidade de $41,7 \%$, enquanto nos previamente com DM a taxa de mortalidade foi de 14,8\%; portanto, 3 vezes maior ${ }^{10}$.

Outra pesquisa realizada na China avaliou 44.672 casos da Covid-19 e a taxa de letalidade foi de $2,3 \%$ (1.023 mortes), sendo elevada entre os sujeitos com doenças crônicas: 10,5\% para aqueles com doença cardiovascular, 7,3\% para casos com DM, 6,3\% para aqueles com doença respiratória crônica, 6,0\% para casos com hipertensão arterial e 5,6\% para câncer ${ }^{11}$.

Além disso, é relevante ressaltar que o DM na vida do sujeito adoecido traz repercussões importantes, tais como ajustamento dos planos subjetivos (significados, identidades) e objetivos (manejo da doença), que se relacionam em um jogo constante de ajustes relacionados ao seu modo de viver ${ }^{12,13}$. 
A relação dos sujeitos adoecidos e suas práticas de cuidado são complexas, pois requerem ações de prevenção e tratamento da doença ${ }^{14}$. Verifica-se, com o advento das infecções da Covid-19, que há uma constante disseminação de informações quanto ao estilo de vida e comportamentos considerados saudáveis. Essas informações podem ser difundidas pelos profissionais de saúde nos momentos das consultas e atividades educativas, mas, também, pela mídia, que se utiliza de dispositivos como televisão e jornais para divulgação de notícias relacionadas à saúde ${ }^{14}$.

A respeito da mídia, seus interesses pelas questões relacionadas à saúde (como o DM e a Covid-19) são progressivamente mais evidentes. São constantes os programas de televisão, as matérias de jornais e sites com diversas informações sobre dietas, medicamentos e epidemia. Esse fascínio pela saúde apresentase, também, pela elaboração de seções fixas dedicadas ao tema, assim como pela prática de contratar profissionais da saúde como especialistas. Os assuntos de saúde, tendo se tornado importantes em nossa sociedade, adquirem valor-notícia, sendo explicitamente reconhecidos pelos profissionais da área, que declaram ser um dos assuntos de maior demanda por parte dos consumidores de produtos midiáticos ${ }^{15}$.

Além disso, é relevante o papel dos meios de comunicação para a divulgação científica e a relação que o sujeito pode estabelecer com os serviços de saúde, pois a mídia, ao facilitar o compartilhamento de saberes, utilizando elementos culturais da sociedade, colabora na prevenção de doenças e na promoção da saúde, à medida em que estimula mudanças na vida dos sujeitos. Utilizar estratégias para captura da atenção dos indivíduos de um determinado grupo, a partir do conhecimento da realidade, da vida cotidiana do sujeito, de suas necessidades, comportamento, interação e expectativa social, demonstra ser uma estratégia para estimular a promoção da saúde de uma forma mais integrada às necessidades e potencialidades da população ${ }^{16}$.

O telejornalismo, por exemplo, cria uma relação pedagógica com a audiência ${ }^{17}$, pois "ensina como se portar diante do texto televisivo, com que atitude comunicativa e em que condições devem aprender as características do gênero"18 (p. 80). Mais do que isso, ao ensinar a se comportar, agir e até pensar, estrutura sua mensagem de uma forma que ela possa ser acessível, com sinais de abertura para interpretação e compreensão e, também, para a internalização e a formação de representações sociais que irão circundar e retroalimentar hábitos, ações e maneiras de enxergar a vida ${ }^{19}$.

Reflexivo com a função social desempenhada pelos meios de comunicação, Bezerra ${ }^{20}$ argumenta que a mídia tem investido em espaços para a discussão dos temas relacionados à saúde. Esse fato se dá pela associação do adoecimento vivenciado pela população a partir dos hábitos adotados, tendo como consequência debates acerca dos cuidados preventivos e curativos em saúde. Além disso, a mídia tem assumido o papel de remédio social para educar as pessoas a cuidarem dos seus corpos.

Há de se considerar que, mesmo a mídia atuando como um importante dispositivo para divulgação de informações sobre o DM, fazem-se necessárias a problematização, a reflexão e a avaliação dessas informações quanto à qualidade, à abrangência e à pertinência dos assuntos tratados, bem como de seus desdobramentos na sociedade ${ }^{14}$.

Dessa forma, considerando a pluralidade de assuntos e abordagens midiáticas sobre a Covid-19 e a complexidade da doença, este estudo tem como objetivo analisar, a partir do referencial teórico da Análise Institucional, os vídeos veiculados sobre o DM e a Covid-19 pela mídia televisiva da Rede Globo de Televisão.

\section{METODOLOGIA}

Foi realizado um estudo documental de abordagem qualitativa. O material pesquisado foi composto por todos os vídeos relacionados ao DM e Covid-19 nos programas da Rede Globo de Televisão no período de 31 de dezembro de 2019 a 31 de maio de 2020, lapso temporal que considera os primeiros casos da doença na China até o início desta pesquisa. 
A TV Globo foi criada em 1965 na cidade do Rio de Janeiro-RJ, sendo o grupo que dirige a emissora denominado Organizações Globo, que também atua em outros ramos da comunicação. Ao todo, são cinco emissoras próprias e 118 afiliadas $^{21}$. Sendo que, no ano de 2019, continuou sendo a emissora de maior audiência com 34,6\% dos televisores ligados e manteve a média de 15,7 pontos de audiência, no mesmo ano, entre $7 \mathrm{~h}$ e $\mathrm{o} \mathrm{h}{ }^{22}$.

Dessa maneira, foi realizada um levantamento retrospectivo dos vídeos do site da Rede Globoi. Este site possui acesso gratuito e disponibiliza vídeos editados de episódios dos programas, com seus respectivos temas e datas, de acordo com a palavra-chave que é utilizada no campo de busca. Os descritores utilizados foram: coronavírus, Covid-19 e diabetes mellitus. A escolha desses descritores se deu por serem considerados, a partir da visualização preliminar dos vídeos sobre o assunto, relevantes para o tema proposto, pois são eles que nomeiam a temática nos meios de comunicação televisivos. Não houve exclusão de nenhum vídeo, pois todos os encontrados sobre DM e Covid-19 traziam o assunto como temática principal.

Os vídeos coletados foram assistidos de forma minuciosa e classificados seguindo as variáveis sobre data de exibição (dia, mês e ano), tema do vídeo (título do vídeo), duração do vídeo, região do Estado Brasileiro a que o programa estava vinculado (nordeste, sudeste, centro-oeste, sul, norte e/ou nível nacional), presença de recursos didáticos/visuais (presença de algum recurso que auxiliava na compreensão do assunto, como tabelas, gráficos, entre outros), presença de algum tipo de serviço agregado (informações que auxiliem o telespectador na efetivação das informações prestadas ou na retirada de dúvidas, como telefones, sites ou endereços), citação do Sistema Único de Saúde (SUS), fontes utilizadas (profissional de saúde, usuários etc.) e abordagem sobre o nível de prevenção quanto ao DM e Covid-19 $9^{23}$. As variáveis foram computadas em um banco de dados no programa Statistical Package for the Social Sciences (SPSS) 21.0 for Windows, e seu tratamento se deu por análise descritiva, visando a caracterização dos vídeos.

Em seguida, todas as falas dos vídeos foram transcritas na íntegra, realizada a leitura acurada de todo o material e análise das informações veiculadas pela mídia televisiva sobre o DM e Covid-19, seguindo o referencial teórico da Análise Institucional.

A análise institucional é uma das correntes do movimento institucionalista que foi proposto por René Lourau e George Lapassade no final da década de 1960 na França e introduzida no Brasil a partir de $1970^{24,25}$. Ela permite a análise das relações de poder que o jogo social mantém com o sistema manifesto e oculto das instituições ${ }^{26,27}$.

As instituições são árvores de decisões lógicas que regulam as atividades humanas, indicando o que é proibido, permitido e indiferente. As instituições compreendem um movimento que a gera (instituinte), um resultado (instituído) e um processo (institucionalização). Para realizar concretamente sua função reguladora, as instituições materializam-se em organizações ou estabelecimentos ${ }^{28}$, como a mídia, por exemplo.

Compreende-se que a análise institucional oferece um arcabouço teórico - como os conceitos de não dito, atravessamentos, grupo sujeito, implicação, discurso, entre outros - que permite explicar como os objetos de estudo são elaborados, se movimentam e transformam segundo um panorama político, social, cultural e econômico no contexto do qual estão inseridos, constituindo construções sociais. Os conceitos trabalhados pela análise institucional oferecem um subsídio que permite auxiliar a visualização dos modos de funcionamento de uma dada realidade social, promovendo um movimento de complexificação das análises e das intervenções ${ }^{29}$.

i Disponível em: https://globoplay.globo.com/. 


\section{RESULTADOS E DISCUSSÃO}

\section{Caracterização dos vídeos}

No período proposto para a pesquisa, foram selecionados 18 vídeos com duração média de 3 minutos, sendo que a distribuição temporal apontou que 50\% $(n=9)$ dos materiais foram divulgados nos meses de março, e o restante no mês de abril, sendo as segundas-feiras o dia da semana com maior número de vídeos com o total de $33 \%(n=6)$.

Com relação à abrangência dos vídeos divulgados, prevaleceram os programas de âmbito nacional e da região nordeste, com $28 \%$ dos vídeos $(n=5)$ cada um. A região sul não apresentou nenhum vídeo sobre o assunto em seus programas estaduais e regionais.

Quanto aos recursos didáticos e visuais, 39\% dos vídeos $(\mathrm{n}=7)$ apresentaram algum recurso, como tabelas, gráficos e infográficos. Tais recursos são importantes porque eles têm a finalidade de ajudar o telespectador a assimilar da melhor forma a mensagem compartilhada ${ }^{30}$.

Quanto aos serviços agregados, $11 \%$ dos vídeos $(\mathrm{n}=2)$ apresentaram essa variável, a saber, informação sobre um serviço de atendimento ao sujeito com DM no município de Vila Velha-ES. Outro vídeo apresentou detalhes de como o sujeito pode obter informações verídicas utilizando o site da Sociedade Brasileira de Diabetes. A apresentação desses serviços agregados ao vídeo é importante, porque disponibiliza explicação sobreórgãos que possam auxiliar o telespectador na efetivação das informações prestadas ou no esclarecimento de dúvidas, fazendo com que os programas de televisão cumpram a função de utilidade pública.

Quanto ao nível de prevenção da Covid-19, 72\% dos vídeos (n=13) apresentaram ações para nível de prevenção primária ${ }^{23}$ para a doença, ou seja, basearam-se na promoção da saúde e prevenção de doenças, como, por exemplo, higienização das mãos e objetos, uso de máscaras, distanciamento social, entre outros.

Em relação ao nível de prevenção relacionada ao DM, prevaleceram $89 \%$ dos vídeos $(n=16)$ com recomendações voltadas à prevenção secundária ${ }^{23}$, isto é, diagnóstico, tratamento e limitações das complicações, como por exemplo, continuar fazendo o uso de medicação, atividades físicas e alimentação saudável, bem como, aferição dos índices glicêmicos no sangue.

Com relação à citação do SUS, nenhuma fala dos sujeitos nos vídeos fez menção à sigla ou ao termo ‘Sistema Único de Saúde', e não foi observado nas imagens o uso do logotipo do SUS em nenhum estabelecimento de saúde mostrado ou vestimentas utilizadas pelos profissionais de saúde entrevistados. Esta é uma variável importante de se observar, pois há de se destacar que a mídia pode atuar como importante ferramenta para a visibilidade das ações do SUS.

Uma análise de 129 matérias sobre DM na mídia impressa no estado do Espírito Santo, por exemplo, constatou que 92,2\% ( $n=119$ ) não citaram o SUS em seus textos, o que evidenciou uma invisibilidade do sistema de saúde relacionado a essa enfermidade ${ }^{14}$. Em outro estudo sobre os vídeos de saúde divulgados pelo Jornal Nacional da Rede Globo, no lapso temporal de 12 meses, os autores constataram que hospitais públicos com falta de profissionais, falhas graves ou negligência eram claramente associados pela mídia ao 'Sistema Público de Saúde' ou ao SUS. Já em notícias relacionadas às campanhas de vacinação, por exemplo, com ações de sucesso, o SUS tendia a ficar à sombra, e a invisibilidade do sistema aumentava ainda mais em ações de promoção da saúde ${ }^{31}$.

Em se tratando do momento de pandemia da Covid-19, a mídia deveria estar mais atenta às ações do SUS, uma vez que diariamente, no início da pandemia, a equipe do Ministério da Saúde fazia pronunciamento, vestidos com coletes do SUS, reforçando o fato de que os cidadãos estavam sob o comando das ações do Ministério e dependentes dos leitos públicos para gestão da pandemia ${ }^{32}$, além das ações da vigilância em saúde, Atenção Primária à Saúde (APS), entre outros. 
Sobre as fontes citadas - organizações ou pessoas que fornecem informações sobre fatos e assuntos de interesse na mídia -, prevaleceram as publicações que citaram os profissionais de saúde (médicos) e usuários com $55 \%$ dos vídeos ( $\mathrm{n}=10)$ e $28 \%(\mathrm{n}=5)$ com apenas os profissionais (médicos) como fonte.

As fontes nas informações divulgadas pela mídia são importantes, pois dão maior relevância sobre o assunto abordado. Em se tratando de temáticas da área da saúde, são os profissionais de saúde que conferem credibilidade às informações prestadas ${ }^{14,33}$.

\section{Diabetes mellitus e Covid-19: uma discussão em pauta}

Por conformar-se como uma doença crônica, o DM possui singularidades na relação dos sujeitos e suas práticas de cuidado em saúde, compreensão com o corpo e as políticas públicas de saúde, com ações de promoção, prevenção e tratamento da doença ${ }^{14}$. No entanto, além de todas as exigências com o gerenciamento do DM - alimentação saudável, exercício físico e uso de medicação -, o sujeito se vê, atualmente, em uma constante imposição para práticas de cuidados em saúde, enfatizada pelo surgimento da Covid-19 e enunciadas pelos discursos - entendido como umas das formas de prática dos agentes, capaz de, por meio da ação, transformar a realidade ${ }^{28}$-, dos profissionais de saúde sobre o assunto.

Todo o processo de cuidado com o DM e medidas preventivas para Covid-19 é extremamente potencializado pela midiatização da sociedade, no contexto da experiência com a saúde. Muitas vezes, a chegada do sujeito ao especialista (profissional de saúde) é determinada pela leitura de textos informativos de diferentes características (leigos, científicos ou jornalísticos), provenientes de diversos suportes (impressos, televisivos ou virtuais) ${ }^{15}$. Entretanto, essas informações necessitam ser discutidas, pois muitos desses textos ou vídeos são entremeados por não ditos.

O não dito, na análise institucional, refere-se às informações que estão omitidas ou deturpadas nos discursos, textos, comportamentos ou outras formas de manifestação. É apontado como fonte de equívocos e conflitos que influenciam a convivência ou, portanto, as causas ou os resultados de um desconhecimento cuja superação se supõe enriquecedora ${ }^{28}$.

Por meio do resgate dos vídeos informativos sobre DM e Covid-19, foi possível aferir que a mídia televisiva se apresenta como um agenciamento capaz de disseminar informações com sentidos diversos para os telespectadores adoecidos ou não.

Um agenciamento (ou dispositivo) é um artifício que produz inovações e devires, remodela virtualidades e inventa o novo radical. Os agenciadores - como a mídia televisiva - "produzem realidades alternativas e revolucionárias que transformam o horizonte considerado do real, do possível e do impossível”28 (p.147). Para Deleuze e colaboradores ${ }^{34}$, um agenciamento é precisamente uma multiplicidade, que, em determinado momento, se comporta como linhas de segmentaridade duras e binárias e, em outro, como linhas moleculares de fuga e de declive.

Sobre esse aspecto, os vídeos sobre DM e a Covid-19 veiculados na televisão demonstram a divulgação de realidades alternativas e revolucionárias com relação às práticas de saúde e novas opções para que os sujeitos com DM mantenham o seu gerenciamento adequado com a doença em tempos de pandemia da Covid-19, como, por exemplo, as consultas médicas a distância e números de telefone para orientações sobre a doença:

[...] nós estamos estabelecendo também, no nível central, com a central de regulação, um plantão de endocrinologistas, onde eles vão fazer uma tele orientação para pacientes e para os profissionais da atenção básica de saúde dos diversos municípios. Um telefone que será disponibilizado para que possa ser feita essa tele orientação para os pacientes diabéticos e que a gente sabe que também são suscetíveis as outras infecções (Médica, Jornal da Manhã, 23/03/2020). 
[...] existem outros meios também como a telemedicina que foi aprovada pelo CFM. Então, a gente pode estar usando outros meios para poder ajudar essas pessoas (Médica, Jornal Anhanguera, $1^{\text {a }}$ Edição, 21/04/2020).

Dessa maneira, de acordo com a divulgação televisiva sobre o serviço de telessaúde para sujeitos com DM, frisa-se que a pandemia da Covid-19 impôs mudanças no delineamento legal que rege a prática de atendimento de saúde a distância, levando o Conselho Federal de Medicina a reconhecer o uso de ferramentas de telemedicina por profissionais médicos com a finalidade de orientar, encaminhar e monitorar pessoas suspeitas ou positivas para o coronavírus ${ }^{35}$. Com base nisso, o Ministério da Saúde regulamentou o uso de telemedicina, excepcionalmente para o contexto da epidemia da Covid-19, com intuito de prestação de abordagem em pré-clínica, suporte assistencial, consulta, monitoramento e diagnóstico em todo o sistema de saúde brasileiro ${ }^{36}$. Essa foi questão fundamental para que o teleatendimento passasse a compor oficialmente o protocolo de manejo clínico desse problema no contexto da $\mathrm{APS}^{37}$, o que resultou, entre outras considerações, na adoção por estados e municípios de centrais de teleatendimento como componentes essenciais de primeiro contato das pessoas com a rede de atenção à saúde ${ }^{38}$.

A implementação adequada dessas tecnologias aumenta a capacidade de atendimento dos serviços de saúde e diminui a dificuldade de acesso das pessoas a orientações qualificadas. Entretanto, tais tecnologias não devem operar apenas como um serviço exclusivo deste período de enfrentamento à Covid-19, pois devidamente implementadas na APS, por exemplo, podem levar a resultados positivos em termos de acesso, resolubilidade, integralidade e abrangência de cuidados, comodidade para as pessoas e qualificação do monitoramento de doenças crônicas já acompanhadas nas unidades de saúde ${ }^{38}$, como o DM.

Quando a mídia televisiva coloca em pauta os serviços de telessaúde para os sujeitos com DM em tempos de pandemia da Covid-19, ela auxilia na criação de estratégias que viabilizam a efetivação do SUS, pois a comunicação e saúde, pensada na perspectiva do direito à comunicação, potencializa a participação e autonomia dos sujeitos, e confere visibilidade às necessidades e demandas de saúde, o que contribui diretamente para o direito à saúde ${ }^{39}$.

Além do mais, essa participação dos sujeitos telespectadores no processo noticioso da mídia televisiva também foi observado nos vídeos analisados, pois na maior parte das informações divulgadas era utilizada uma abordagem de perguntas e respostas enviadas pelos telespectadores aos profissionais de saúde entrevistados:

Nós vamos continuar falando das pessoas que vivem com diabetes e como elas podem se proteger da COVID-19. Você tem alguma dúvida sobre o assunto, participa com a gente. É só mandar sua pergunta para WhatsApp (Apresentador, Jornal Anhanguera, $1^{\text {a }}$ Edição, 21/04/2020).

São 2 horas e 30 minutos de jornal, mas só com as perguntas que eu recebi no WhatsApp dá para gente ficar muito tempo, mas pelo menos deu para tirar algumas dúvidas bem importantes (Repórter, Bom dia Ceará, 30/03/2020).

Vamos para o nosso telão, porque já recebemos algumas perguntas. Pergunta corona, aqui para os nossos entrevistados (Apresentador, Combate ao Coronavírus, 13/04/2020).

A inserção do público telespectador no processo noticioso, vista como um jornalismo participativo, configura uma nova dinâmica da rotina dos profissionais jornalistas. No caso dos telejornais, estes deixam de assumir uma comunicação exclusivamente unilateral, de um para muitos ${ }^{40}$, passando a incluir os telespectadores na dinâmica de produção noticiosa, mesmo com limitações, uma vez que cabe aos jornalistas 
verificar as informações (ou as perguntas) recebidas ${ }^{41}$. Além do mais, a convergência tecnológica (inclusão da Web e de mídias móveis na cadeia de distribuição de notícias ${ }^{42}$ ) vem potencializando a interação com o público, favorecendo o surgimento de novos modelos de produção jornalística, com a proliferação dos recursos característicos das mídias digitais, tais como distribuição multiplataforma ${ }^{43}$.

Os telespectadores inseridos na produção de matérias na mídia televisiva, especialmente na área da saúde, podem ser denominados Grupo Sujeito, ou seja, um grupo que se constitui uma utopia ativa, capaz de gerar suas próprias normas - como realização de pautas com assuntos de sua escolha ou empreendimento de perguntas ao entrevistado fazendo com que mudem os rumos da entrevista. Assim, eles deixam de ser grupo sujeitado, isto é, aquele alienado em objetivos, procedimentos e normas que lhes são impostas, como por exemplo, assistir de forma passiva a uma matéria de televisão sem poder elucidar as dúvidas sobre o assunto discutido ${ }^{28}$.

É importante ressaltar que a participação do cidadão no processo noticioso, que fortalece o jornalismo participativo, pode colocar em evidência os atravessamentos e potencialidades sobre a experiência individual e coletiva de se viver com o DM em tempos de pandemia da Covid-19.

Um atravessamento é um entrelaçamento e articulação de orientação conservadora, que serve de exploração e dominação ${ }^{28}$ dos grupos sociais, como sujeitos com DM. Dessa forma, pode-se notar pelos vídeos analisados que a falta de medicação para o DM e a disseminação de notícias falsas, são alguns dos atravessamentos vivenciados pelos sujeitos atualmente, apresentados na televisão:

Olha quem tem diabetes está dentro do grupo de risco para o novo coronavirus, mas um remédio importante para o controle do diabetes está em falta aqui na rede pública do DF (Apresentador, DF1, 24/03/2020).

[...] ele é um remédio muito caro... nem é todo mundo que tem acesso. E para nós, ficamos procurando de posto em posto, ficamos expostos a contrair o coronavírus (Usuária, DF1, 24/o3/2020).

Com toda sua atenção voltada para o novo coronavirus outras doenças estão sendo deixadas de lado. Pacientes com diabetes reclamam que estão há seis meses sem receber da prefeitura de Belém material para exames diários (Apresentador, Bom dia Pará, 19/03/2020).

[...] Existem umas fake news dizendo que a gente deve parar de tomar medicação para diabetes e isso é mentira, não devemos parar (Médico, Bem Estar, 21/03/2020).

A menor atenção aos sujeitos com doenças crônicas, como o DM, apresentada pela falta de medicação para a doença e envolvimento maior dos serviços de saúde com a Covid-19, pode ser justificada, entre outras questões, pela fragilidade de se fazer um planejamento nos serviços, o que conduz a uma valorização das ações voltadas para doenças infecciosas, em detrimento do desenvolvimento das ações para doenças crônicas. Além disso, Mendes ${ }^{44}$ acrescenta que as condições de saúde, com forte predomínio das doenças crônicas, não podem ser respondidas com eficiência, efetividade e qualidade por sistemas de saúde voltados, predominantemente, para as condições agudas e para as agudizações das doenças crônicas e estruturados de forma fragmentada.

Essa fragmentação nos serviços de saúde traz como consequência uma atenção centrada no cuidado profissional, especialmente o médico, uma ênfase nas intervenções curativas e reabilitadoras e inexistência da continuidade da atenção $0^{45}$, o que gera também uma dificuldade das ações de educação popular em saúde que tem como objetivo ampliação do diálogo com os sujeitos com informações precisas e necessárias para atenção às doenças crônicas, reveladas pelo destaque às notícias falsas sobre o assunto. 
Dessa forma, atualmente, conforme fragmentos anteriores, tem-se percebido que o aumento de divulgação de informações equivocadas, denominadas fake news, tem se configurado como uma ferramenta que gera preocupação dos profissionais de saúde, dúvidas entre os sujeitos adoecidos e enormes desafios para o sistema de saúde.

As fake news são informações/notícias/postagens elaboradas de forma inverossímil que, sem a devida averiguação, levam o indivíduo a pseudoinformações ${ }^{46}$. Em um estudo realizado com 70 fake news sobre a Covid-19 no Brasil, foi observado que esses conteúdos se disseminam de forma rápida, expondo a população à propagação de condutas inadequadas, principalmente quando as evidências científicas passaram a ser questionadas no campo da política por alguns governantes ${ }^{47}$. Assim posto, o compartilhamento das fake news é apontado como uma das principais razões para a não aceitação de medidas preventivas e de cuidados estabelecidos pela ciência em prol da saúde pelo mundo ${ }^{48}$.

Além dos atravessamentos, a participação dos sujeitos no processo noticioso também tem dado ênfase às potencialidades vivenciadas pelos indivíduos com DM em tempos de pandemia da Covid-19. Uma potência se refere às capacidades atuais ou virtuais de se produzir, transformar ou inventar, ou seja, designa a magnitude das forças geradoras do radicalmente novo ${ }^{28}$, como um caso relatado em uma reportagem sobre a união de uma família para fazer exercícios físicos em casa e deixar o mau humor de lado em tempos de distanciamento social:

Em Bebedouro-SP uma família cortou o mau humor da quarentena e tá usando o isolamento em casa para fortalecer a saúde (Apresentador, Bom dia Cidade - Campinas/Piracicaba, 16/04/2020).

[...] Desde o começo da quarentena a casa da dona I. e do marido virou uma grande academia. A ideia foi do filho que é educador físico (Repórter, Bom dia Cidade - Campinas/Piracicaba, 16/04/2020).

Com isolamento percebemos que em casa daria para fazer um monte de coisa. Nós trabalhamos força, flexibilidade, resistência, equilíbrio, só com materiais que temos praticamente em casa. Já melhorou o controle do diabetes, melhorou todo desenvolvimento das atividades diárias deles e principalmente a gente percebe que os riscos de acidentes domésticos estão caindo muito (Usuário, Bom dia Cidade Campinas/Piracicaba, 16/04/2020).

As notícias sobre as práticas cotidianas, como a atividade física da família mencionada, acontecem por conta da individualização da notícia, ou seja, as reportagens proliferam sobre cuidado, posicionando os temas da saúde com mais frequência na vida rotineira dos sujeitos. Ainda que a dimensão política se faça presente, especialmente, nas discussões sobre sistema público de saúde, o que se tem observado é o crescimento das informações sobre cuidados individuais, por meio de narrativas biográficas que podem aparecer sob forma de testemunhos e denúncias ${ }^{15}$.

Além dos testemunhos dos sujeitos com DM para atividade física, foi observado que a mídia televisiva também expõe os discursos dos profissionais de saúde na 'lógica do risco', ou seja, a presença de uma ameaça constante da doença na sua virtualidade. Dessa forma, o redimensionamento das ideias sobre qualidade de vida trouxe repercussões no processo de subjetivação, pois os indivíduos passaram de seres cotidianamente saudáveis a seres doentes em potencial, ou eventualmente doentes, cuja condição é ignorada, como no caso de uma glicemia elevada que ainda não foi diagnosticada pelo exame, mas já está presente ${ }^{49-52}$.

A saúde, por essa lógica, não é só mais uma finalidade de vida, mas, muitas vezes, infere sentido a ela, e os profissionais de saúde especialistas são permanentemente convocados a ensinar às pessoas como gerenciar seus desejos e corpos e ofertar orientações na gestão do cotidiano ${ }^{53}$, conforme apresentado a seguir: 
Vai tomar todos os cuidados com a alimentação, procurar alimentos muito saudáveis, e tomar os medicamentos, fazendo controle, não deixando de fazer atividade física [...] ter cuidado com a saúde mental, porque tem paciente que é diabético e faz um pânico entre aspas e tem mais um receio porque ele faz parte de um grupo de risco, mas não precisa entrar em pânico, é só cuidar da sua doença e seguir as orientações (Médica, Jornal Anhanguera, $1^{\text {a }}$ Edição - TO, 21/04/2020).

A questão da alimentação adequada [...] priorizar as áreas de alimentos saudáveis [...] com relação aos exercícios é fundamental, né? nessa fase a gente orienta exercício em casa se você já fazia antes, hoje tem inúmeros aplicativos a gente tem visto várias orientações a internet de você pelo menos não ficar realmente parada (Médica, Bom dia Ceará, 30/03/2020).

A privação da responsabilidade pelo cuidado, por uma governabilidade de $\mathrm{si}^{54}$, apresentada pelos especialistas, é abordada nos vídeos sobre o DM e Covid-19. No contexto da sociedade de risco ${ }^{51}$, os discursos dos profissionais na mídia estimulam os sujeitos a fazerem escolhas individuais mais saudáveis, o que promoveria uma vida mais feliz, com aumento da autoestima e da segurança. Entretanto, com o avanço da epidemiologia, o reconhecimento de juízo de valor favorável ou não às rotinas padronizadas como saudáveis produziu uma regularidade no estilo de vida que possibilita a quantificação da vida sem regras, do sedentarismo e a sua associação a um maior risco de doença ${ }^{49}$. Esse processo trouxe algumas consequências, por exemplo, a moralização dos estilos de vida arriscados, conferindo aos sujeitos que fogem às regras o estigma do pecado e da fraqueza de caráter, e a responsabilidade individual, sem considerar as questões macrossociais que potencializam as condutas de risco em prol dos aspectos relativos exclusivamente ao sujeito $^{50}$.

Outrossim, a divulgação midiática sobre os assuntos da saúde, como a Covid-19 e o DM, deve ser explorada para que seja realizada reflexão a respeito do que é disseminado, bem como análise da implicação - lugar que o indivíduo ocupa no mundo ${ }^{55}$-, dos sujeitos no processo de comunicação.

\section{CONSIDERAÇÕES FINAIS}

Na contemporaneidade, a mídia tem se tornado uma relevante estratégia para disseminação de assuntos na área da saúde e, a partir do seu poder de pautar determinadas questões, ela pode influenciar na constituição de crenças, opiniões e condutas sobre diversas temáticas.

A respeito dos cuidados com o DM e Covid-19, em conformidade ao observado nesta pesquisa, a mídia televisiva, por meio do jornalismo participativo e da visibilidade dos discursos dos profissionais de saúde, tem apresentado as medidas preventivas para se evitar contaminação por Covid-19, os esclarecimentos quanto aos serviços ofertados, a importância da alimentação, atividade física e o uso de medicação aos sujeitos com DM.

Entretanto, os profissionais de saúde abordados pela mídia televisiva ainda se utilizam de um discurso voltado para a lógica do risco, na qual se anuncia uma ameaça permanente, partindo da orientação de como a população precisa gerenciar os seus corpos, sem considerar, muitas vezes, os aspectos socioeconômicos que dificultam a produção do cuidado em saúde. Além disso, é notória a invisibilidade do SUS nesse meio de comunicação, quando se percebeu que nenhum vídeo fez menção a esse sistema.

Dessa maneira, a relação dos profissionais de saúde com os meios de comunicação deve ser revista no sentido de disseminar orientações que possam considerar o aspecto macrossocial de se conviver com o DM em tempos de pandemia da Covid-19, para que as ações estejam em concordância com os modos de viver da população. 
Espera-se também que os meios de comunicação - importante dispositivo para potencialização do cuidado em saúde, especialmente em tempos de pandemia da Covid-19 - possam continuar trabalhando para o compartilhamento de informações éticas e alinhadas com as necessidades de saúde, ofertando visibilidade ao SUS.

\section{REFERÊNCIAS}

1. World Health Organization. Coronavirus disease (COVID-19) pandemic. Genebra: The Organization; 2020 [cited 2020 June 13]. Available from: https://www.who.int/emergencies/diseases/novelcoronavirus-2019.

2. Wu C, Chen X, Cai Y, Xia J, Zhou X, Xu S, et al. Risk factors associated with acute respiratory distress syndrome and death in patients with coronavirus disease 2019 pneumonia in Wuhan, China. JAMA Intern Med [Internet]. 2020 July [cited 2020 June 13];180(7):1-11. Available from: https:// jamanetwork.com/journals/jamainternalmedicine/fullarticle/2763184. Epub ahead of print.

3. Ministério da Saúde (BR). Guia de vigilância epidemiológica: emergência de saúde pública de importância nacional pela doença pelo coronavírus 2019: vigilância integrada de síndromes respiratórias agudas doença pelo coronavírus 2019, influenza e outros vírus respiratórios. Brasília: O Ministério; 2020 abr. 03.

4. Sardu C, Gambardella J, Morelli MB, Wang X, Marfella R, Santulli G. Is COVID-19 an endothelial disease? Clinical and basic evidence. Preprints 2020040204 [Preprint]. 2020 [cited 2020 May 12]. Available from: https://www.preprints.org/manuscript/202004.0204/v1.

5. World Health Organization. Preventing chronic diseases: a vital investment. Geneva: The Organization; 2005.

6. Schmidt MI, Duncan BB, Azevedo e Silva G, Menezes AM, Monteiro CA, Barreto SM, et al. Chronic noncommunicable diseases in Brazil: burden and current challenges. Lancet 2011; 377(9781):1949-61.

7. Ministério da Saúde (BR). Estratégias para o cuidado da pessoa com doença crônica: diabetes mellitus. Brasília: O Ministério; 2013.

8. Oliveira JEP, Vencio S, organizadores. Diretrizes da Sociedade Brasileira de Diabetes: 2015-2016. São Paulo: AC Farmacêutica; 2016.

9. Internacional Diabetes Federation. Atlas de la diabetes de la FID. 9th ed. Bruxelas: La federación; 2019.

10. Bode B, Garrett V, Messler J, McFarland R, Crowe J, Booth R, et al. Glycemic Characteristics and Clinical Outcomes of COVID-19 Patients Hospitalized in the United States. J Diabetes Sci Technol [Internet]. 2020 July [cited 2020 May 12];14(4):813-21. Available from: https://glytecsystems.com/wpcontent/uploads/JDST-Glytec-Covid-Research.pdf.

11. Wu Z, McGoogan JM. Characteristics of and Important Lessons From the Coronavirus Disease 2019 (COVID-19) Outbreak in China: Summary of a Report of 72314 Cases From the Chinese Center for Disease Control and Prevention. JAMA. 2020 Apr 7;323(13):1239-42.

12. Thoolen B, De Ridder D, Bensing J, Gorter K, Rutten G. No Worries, no impact? A systematic review of emotional, cognitive, and behavioural responses to the diagnosis of type 2 diabetes. Health Psychol Rev. 2008;2(1):1-29.

13. Barsaglini RA. As representações sociais e a experiência com o diabetes: um enfoque socioantropológico. Rio de Janeiro: Fiocruz; 2011.

14. Coqueiro JM, Oliveira AE, Figueiredo TAM. Diabetes Mellitus na mídia impressa: uma análise das matérias nos jornais do Espírito Santo, Brasil. Saúde Debate 2019;43(121):530-42.

15. Lerner K. Doença, mídia e subjetividade: algumas aproximações teóricas. In: Lerner K, Sacramento I, organizadores. Saúde e jornalismo: interfaces contemporâneas. Rio de Janeiro: Fiocruz; 2014. p. 15161.

16. Machado AS, Silva VC. Além de informar: a comunicação social a serviço dos processos de promoção em saúde e das redes de gestão e atenção. R Eletr Com Inf Inov Saúde [Internet]. 2012 ago. [citado em 2020 set. 07];6(2 supl):1-11. Disponível em: https://www.reciis.icict.fiocruz.br/index.php/reciis/article/ view/760/1402. 
17. Vilches L. Manipulación de la información televisiva. Barcelona: Paidós; 1989.

18. Vizeu A. O telejornalismo como lugar de referência e a função pedagógica. Rev Famecos. 2009;16(40):77-83.

19. Silva LJC, Pereira Júnior AEV. Os saberes da pedagogia no telejornalismo: Paulo Freire e a prática jornalística. Rev Famecos. 2019;26(1):1-18.

20. Bezerra HPO. Educação em saúde e mídia: uma proposta na "medida certa". Praxia [Internet]. 2013 [citado em 2020 set. 06];1(2):544-58. Disponível em: https://www.revista.ueg.br/index.php/praxia/ article/view/1414/940.

21. Tv Globo [Internet]. Rio de Janeiro: Grupo Globo; 2015 [citado em 2020 set. 03]. Disponível em: https://web.archive.org/web/20151003075529/http://grupoglobo.globo.com/tv globo.php.

22. Globo cai e Record sobe nos índices de audiência em 2019. Pleno News [Internet]. 2019 nov. 18 [citado em 2020 abr. 28];Entretenimento. Disponível em: https://bit.ly/314xFij.

23. Leavell HR, Clark EG. Medicina preventiva. São Paulo: McGraw-Hill do Brasil; 1976.

24. L'Abbate S. Análise institucional e intervenção: breve referência à gênese social e histórica de uma articulação e sua aplicação à Saúde Coletiva. Mnemosine 2012;8(1):194-219.

25. Figueiredo TAM. A Análise Institucional na formação acadêmica da Pós-Graduação em Saúde Coletiva no Espírito Santo. In: L'Abbate S, Mourão ML, Pezzato LM, organizadoras. Análise institucional e Saúde Coletiva. São Paulo: Hucitec; 2013. p. 607-32.

26. Hess R. O movimento da obra de Lourau (1933-2000). In: Altoé S, organizadora. René Lourau: analista institucional em tempo integral. São Paulo: Hucitec; 2004. p. 15-46.

27. Monceau G. Pratique socianalytiques et sócio-clinique institutionnelle. L'Harmattan. 2003;(147):11-33.

28. Baremblitt G. Compêndio de Análise Institucional e outras correntes: teoria e prática. 6. ed. Belo Horizonte: FGB/IFG; 2012.

29. Kasper M, Fortuna CM, Braghetto GT, Marcussi TC, Feliciano AB, L'Ábatte S. A análise institucional na produção científica em saúde: uma revisão integrativa de literatura. Rev Esc Enferm USP [Internet]. 2020 [citado em 2020 out. 13];54:e03587. Disponível em: https://www.scielo.br/scielo.php?pid=S0080-

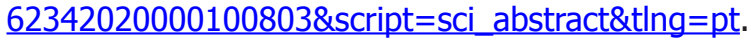

30. Paternostro, VI. O texto na TV: manual de telejornalismo. Rio de Janeiro: Campus; 1999.

31. Lefèvre F, Caron E. A saúde mediada pela televisão: o caso do Jornal Nacional. In: Sacramento I, organizador. Mediações comunicativas da saúde. Rio de Janeiro: Editora Multifoco; 2017. p. 287-303.

32. Corôa R. A remoralização da saúde em tempos de pandemia: uma esperança para o SUS. Dilemas Rev Est Conf Cont Soc 2020;1-14.

33. Coqueiro JM, Cavaca AG, Emerich TB, Antunes MN, Oliveira AE, Figueiredo TAM. Diabetes mellitus na mídia: uma proposta de protocolo de coleta e classificação de dados para pesquisa. Rev Bras Pesq Saúde 2018;20(2):74-87.

34. Deleuze G, Parnet C. Diálogos. São Paulo: Escuta; 1998.

35. Conselho Federal de Medicina (BR). Ofício CFM no 1756/2020 - COJUR [Internet]. Brasília, DF: O Conselho; 2020 [citado em 2020 abr. 14]. Disponível em: http://portal.cfm.org.br/images/PDF/2020 oficio telemedicina.pdf.

36. Portaria MS/GM no 467 (BR), de 20 de março de 2020. Dispõe, em caráter excepcional e temporário, sobre as ações de Telemedicina, com o objetivo de regulamentar e operacionalizar as medidas de enfrentamento da emergência de saúde pública de importância internacional previstas no art. $3^{\circ}$ da Lei no 13.979, de 6 de fevereiro de 2020, decorrente da epidemia de COVID-19. DOU [Internet]. 2020 mar. 21 [citado em 2020 jun. 14]. Disponível em: https://www.in.gov.br/en/web/dou/-/portaria-n-467-de-20de-marco-de-2020-249312996.

37. Ministério da Saúde (BR). Protocolo de manejo clínico do coronavírus (Covid-19) na Atenção Primária à Saúde: versão 07 [Internet]. Brasília, DF: O Ministério; 2020. [citado em 2020 nov. 05]. Disponível em: https://saude.rs.gov.br/upload/arquivos/202004/14140606-4-ms-protocolomanejo-aps-ver07abril.pdf. 
38. Sarti TD, Lazarini WS, Fontenelle LF, Almeida APSC. Qual o papel da Atenção Primária à Saúde diante da pandemia provocada pela COVID-19? Epidemiol Serv Saúde [Internet] 2020 [citado em 2020 out. 13];29(2): e2020166. Disponível em: https://www.scielo.br/scielo.php?script=sci arttext\&pid $=\mathrm{S} 2237-96222020000200903$.

39. Guareschi P. O direito humano à comunicação: pela democratização da mídia. Petrópolis: Vozes; 2013.

40. Gillmor D. Nós, os media. Lisboa: Editorial Presença; 2004.

41. Frazão SM, Brasil A. A participação do telespectador na produção da notícia em telejornal: transformação do processo noticioso e da rotina profissional. Braz Journalism Res 2013;9(2):112-29.

42. Belochio V. Considerações sobre a distribuição multiplataforma e suas afecções nos contratos de comunicação propostos em Zero Hora. Est Jornalismo Mídia. 2015;12(1):29-42.

43. Gomes I. Metodologia de análise de telejornalismo. In: Gomes Itania, organizadora. Gêneros televisivos e modos de endereçamento no telejornalismo. Salvador: EDUFBA; 2011.

44. Mendes EV. As redes de atenção à saúde. Ciênc. Saúde Coletiva 2010;15(5):2297-2305.

45. Fernandez J. Los sistemas integrados de salud: un modelo para avanzar tras completar las transferencias. Barcelona: B\&F Gestión y Salud; 2004.

46. Allcott H, Gentzkow M. Social media and fake news in the 2016 election. J Econ Perspect. 2017;31(2):211-16. doi: https://doi.org/10.1257/jep.31.2.211.

47. Neto M, Gomes TO, Porto FR, Rafael RMR, Fonseca MHS, Nascimento J. Fake news in the context of the covid-19 pandemic. Cogitare Enferm [Internet]. 2020 [cited 2020 Oct 13];25:e72627. Available from: https://revistas.ufpr.br/cogitare/article/view/72627/pdf.

48. Monari ACP, Bertolli Filho C. Saúde sem fake news: estudo e caracterização das informações falsas divulgadas no canal de informação e checagem de fake news do Ministério da Saúde. Rev Mídia Cotidiano 2019;13(1):161-86.

49. Bagrichevsky M, Castiel LD, Vasconcellos-Silva PR, et al. Discursos sobre comportamento de risco à saúde e moralização da vida cotidiana. Ciênc Saúde Colet. 2010;15(1):1699-708.

50. Castiel L, Guilam MCR, Ferreira MS. Correndo o risco: uma introdução aos riscos em saúde. Rio de Janeiro: Fiocruz; 2010.

51. Beck U. Sociedade de risco: rumo a uma outra modernidade. 2. ed. São Paulo: Ed. 34; 2011.

52. Vaz P. As narrativas midiáticas sobre cuidados com a saúde e a construção da subjetividade contemporânea. Logos 2006;25(1):85-95.

53. Sodré M. A narração do fato: notas para uma teoria do acontecimento. Petrópolis: Vozes; 2009.

54. Lerner K, Sacramento I. Apresentação. In: Lerner K, Sacramento I, organizadores. Saúde e Jornalismo: Interfaces contemporâneas. Rio de Janeiro: Fiocruz; 2014. p. 15-31.

55. Lourau R. Análise institucional e prática de pesquisa. Rio de Janeiro: UERJ; 1993. 